\title{
Evidence of vertical transmission of Novel Duck Orthoreovirus in ducks
}

\author{
Hongzhi Wang ${ }^{1}$, Yuanyuan Wang $^{2}$, Bin $\mathrm{Gao}^{3}$, shuai Zhang ${ }^{3}$, YOU XIANG DIAO ${ }^{3}$, and YI \\ TANG $^{3}$
}

${ }^{1}$ Shandong Agriculture University

${ }^{2}$ China Animal Health and Epidemiology Center

${ }^{3}$ Shandong Agricultural University

July 20, 2020

\begin{abstract}
Since 2017, duck spleen necrosis caused by a new variant duck orthoreovirus (N-DRV) infection had been observed in many provinces in China. This disease causes the growth and development of ducks to be retarded, and the feed return rate is reduced. Because the target organ of the disease is mainly the spleen, the immune suppression of the diseased ducks is easy to secondary infection of other pathogens. In this study, we successfully constructed a breeding duck artificial infection model and found that N-DRV infection can cause pathological changes such as ovarian hemorrhage, follicle atrophy, and fallopian tube bleeding in breeding ducks, resulting in a significant reduction in the fertilization rate and hatching rate of breeding eggs. The qPCR method was used to detect viral RNA in samples of egg vitelline membrane, duck embryo, and duckling's spleen. It was found that viral RNA was present in the above samples. Although there were no obvious clinical symptoms in the early stage of ducklings, autopsy revealed obvious pathological changes in the spleen and other organs. The sequence distance and phylogenetic analysis of the strain re-isolated from the spleen samples of ducklings (named N-DRV-SD19) confirmed that N-DRV-SD19 was consistent with the strain N-DRV-XT18 used for the infection of the breeding ducks. The findings of the above research confirmed that N-DRV can be vertically transmitted through eggs, which will provide an important reference for the prevention and control of the disease.
\end{abstract}

\section{Introduction}

Avian orthoreovirus (ARV) is non-enveloped double-srtanded RNA (dsRNA) virus, which is classified into the genus Orthoreovirus, familyReoviridae (Jones, 2000). The ARV genome is about 23 kilobase and contains ten dsRNA segments, including three L-class (L1, L2 L3), three M-class (M1, M2, M3) and four S-class (S1, S2, S3, S4), which encode eight structural proteins $(\lambda \mathrm{A}, \lambda \mathrm{B}, \lambda \mathrm{C}, \mu \mathrm{A}, \mu \mathrm{B}, \sigma \mathrm{A}, \sigma \mathrm{B}, \sigma \mathrm{C}$,$) and four$ non-structural proteins ( $\mu$ NS, p10, p17, $\sigma \mathrm{NS}$ ) (Nick et al., 1975; Joklik, 1982; Su et al., 2006). ARV can infect a variety of poultry including chicken (Tang and Lu, 2015a), duck (Malkinson et al., 1981), goose (Vilmos et al., 2003; Yun et al., 2012), pigeon (Vindevogel et al., 1982) and other domestic poultry, and has been associated with a variety of clinical symptoms (Jones and Guneratne, 1984).

In recent years, there have been reports of new genomic variations in these novel ARV strains isolated from vaccinated broiler flocks (Su et al., 2006; Tang and Lu, 2015b; Tang et al., 2015; Hao Chen et al., 2019). Since the first report of Muscovy duck infection with ARVs in China in 1997, waterfowl-derived ARVs have already spread in most parts of China. And in the process of epidemic, mutants with different pathogenic characteristics appeared. The representative strains mainly including a new type of Muscovy orthoreovirus (N-MDRV) that causes necrotic and bleeding spots in the liver of Muscovy ducks, also known as liver white spot disease, DRV that causes hemorrhagic symptoms of multiple organs (Zheng et al., 2016), DRV causes 
hemorrhagic necrotic hepatitis which characterized by liver necrosis and bleeding in infected ducks (Z. Chen et al., 2012), and new type duck orthoreovirus (N-DRV) that causes necrosis in the spleen of Pekin ducks (Zheng et al., 2016). Since the above strains are viruses that have appeared in Chinese duck flocks in recent years, the current research on DRV is far less comprehensive than the chicken-derived ARV. DRV needs to continue in-depth research in terms of virus transmission mode, gene-function relationship, antigen characteristics and so on.

Since 2017, a new epidemic disease characterized by spleen necrosis and swelling has emerged in China's main meat duck breeding provinces, which mainly causes large groups of ducks with poor mental state, appetite loss and even death (Wang et al., 2019). Since the outbreak of the disease, the incidence of this new disease has been rising rapidly and expanding geographically. The mortality rate of infected ducks can reach $50-60 \%$. Due to the necrosis of spleen, the weakened immunity of the body is easy to cause secondary infection, which brings great economic losses (Montgomery et al., 1986). This disease has become one of the major diseases that seriously harm the development of China's duck breeding industry.

Regarding the study of ARV transmission methods, researchers have confirmed that ARV can be transmitted vertically through eggs as early as 1970s (Menendez and Cowen, 1975), and carried out research on the protective effect of maternal antibodies (Heide, 2000). But at the same time, some researchers believe that the rate of transmission through egg is extremely low, less than $2 \%$. Regarding the transmission method of $\mathrm{N}$-DRV, we had determined that healthy ducks can cause infection through ingestion of fecal contaminated water and feed, or direct contact with ducks infected with N-DRV and cause respiratory infections, which shows N-DRV is spread horizontally (Wang et al., 2020). The epidemiological survey results of wild waterfowl show that N-DRV can be detected in the wild waterfowl feces such as wild ducks and swans, indicating that the migration of wild waterfowl may play an important role in the spread of N-DRV. However, there is no definite report on whether N-DRV can spread through vertical transmission.

In order to investigate whether N-DRV can vertically spread in breeding ducks, through experimental infection of healthy breeding ducks during the laying period, a N-DRV artificial infection animal model was successfully established (Chen et al., 2016). Through the hatching of eggs, the detection of pathogens in hatching eggs, embryos, and ducklings, we found that the fertilization and hatching rates of the ducks in the infected group were lower than those in the control group, and the samples of hatching eggs, embryos and ducklings in the infected group were all tested positive for N-DRV. This discovery provides detailed evidence for the vertical transmission of N-DRV and provides an important reference for the prevention and control of the disease.

\section{Materials and methods}

\subsection{Ethics statement}

All animal infection experiments were conducted in accordance with the "Guidelines for Experimental Animals" of the Ministry of Science and Technology (Beijing, China) and were conducted under the supervision of the Animal Protection and Utilization Committee of Shandong Agricultural University.

\subsection{Serological testing of N-DRV}

N-DRV antibody levels in the serum were detected by indirect enzyme-linked immunosorbent assay (ELISA). Briefly, the purified $\sigma \mathrm{C}$ protein $(50 \mathrm{ng} / \mu \mathrm{L}$, prepared in our previous study ) was added into each well of the ELISA plates (NEST Biotechnology, Wuxi, China) and incubated for $12 \mathrm{~h}$ at 4, and then the antigen coating solution in the ELISA plates were completely aspirated, and plates were washed three times with washing buffer ( PBS containing 0.05\% Tween-20, PBST ). Add 200 $\mathrm{LL}$ of 5\% skim milk powder (Solarbio, Beijing, China; w/v) to each well for blocking, and incubate at 37 for $2 \mathrm{~h}$. After blocking, discard the blocking solution and wash 3 times. The duck serum sample (1:10) to be tested was added to the wells. A negative control and a blank control group were set. After incubating at 37 for $1 \mathrm{~h}$, the samples were washed three times with PBS and the washing solution in the wells was blotted. Then, the plates were incubated with rabbit anti-duck immunoglobulin $\mathrm{G}(\mathrm{IgG})$ at $1: 1000$, incubated again for $1 \mathrm{~h}$ at 37 , and were washed three 


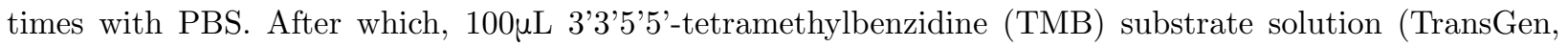
Beijing, China) was added to each plate and left to develop at $37^{\circ} \mathrm{C}$ in the dark for 15 minutes. So far, stop buffer $\left(3 \mathrm{M} \mathrm{H}_{2} \mathrm{SO}_{4}\right)$ was add to stop the reaction at $100 \mu \mathrm{L}$ /well (S. Zhang et al., 2020). The optical density (OD) values at $450 \mathrm{~nm}$ of each sample was measured using an ELISA microplate reader (Thermo Fisher Scientific, Waltham, MA, USA). The cutoff value was an OD of 0.459 at $450 \mathrm{~nm}$. Each sample is repeatedly tested 3 times to ensure the accuracy of the test results.

\subsection{Virus strain and artificial infection test}

The strain N-DRV-XT18 (GenBank Accession No. MK749398-MK749407) used in this study was a variant duck orthoreovirus strain isolated from the spleen of Pekin duck with splenic swelling and necrosis isolated in 2018. LMH (Leghorn Male-chicken Hepatocellular-carcinoma, ATCC CRL-2013) cell was used for virus isolation and proliferation, according to conventional virus cell culture methods (Tang and Lu, 2015a). The median tissue culture infective dose $\left(\mathrm{TCID}_{50}\right)$ of the N-DRV-XT18 strain was $10^{-6.52} / 0.1 \mathrm{~mL}$, which was calculated using the Reed-Muench assay. Before the inoculation, the virus culture supernatant was tested for purity by PCR to exclude other pathogenic contamination (Wang et al., 2020). (The sequence of the primers was not shown).

60 healthy female and 15 male breeding ducks (35-weak-old) were purchased from a breeding farm in Tai'an city, Shandong province. Before the experiment, each duck collected serum samples and cloacal swabs, and tested by ELISA and qPCR to confirm that its N-DRV antibody test was negative, and it did not contain N-DRV infection or other duck pathogens. These ducks were randomly divided into three groups, orally administered group, intramuscular injection group and control group, each group of 20 female ducks and 5 male ducks. The orally administered and intramuscular injection group were inoculated with $0.2 \mathrm{~mL}$ $\left(10^{-6.52} / 0.1 \mathrm{~mL}\right)$ virus culture supernatant. The control group was inoculated with equal doses of strokephysiological saline solution (SPSS) at the same injection site (Wang et al., 2020). The three groups were reared in separate houses and ensured that the feeding conditions were consistent and free from contamination by external pathogens.

\subsection{Samples collection}

The eggs from the ducks of the experimental group and the control group were collected for hatching, and the fertilization rate and hatching rate of each group were counted. Collect eggs as early as possible every day to reduce the time that eggs are stored outdoors. Use a disinfectant to scrub the eggshells to prevent bacterial infections on the eggshells from affecting the hatching, and then place them in the incubator for hatching. On the 6th day of hatching, the yolk membrane was collected from the unfertilized eggs (Wei et al., 2020). The spleen of the dead embryos during the hatching process was collected, and the hatched ducklings also received spleen tissue for virus detection. Finally, samples that test positive are freeze-thawed for virus isolation.

\subsection{Genomic RNA extraction and quantitative real-time PCR detection}

Viral RNA was extracted from the embryo and spleen tissue using RNAprep pure Tissue Kit (TIANGEN, Beijing, China) following the manufacturer's instructions. These extracted RNA samples were detected the concentration using DeNovix DS-11 Spectrophotometer (Nanodrop, USA) and stored at -20. The N-DRV nucleic acid in the sample were detected by the Taqman probe real-time quantitative PCR method previously established by our team, the N-DRV specific primers and TaqMan probe were designed to target the $\mathrm{S} 2$ gene of N-DRV strain, and the smallest detectable amount was 10 copies $/ \mu \mathrm{L}$. The primers and probe sequences were shown as follows: N-DRV-S1-F: 5'-ATGGATCGCAACGAGGTGATAC-3' (958-978), N-DRVR: 5'-CTAGCCCGTGGCGACGGT-3' (1022-1042), Probe: 5'-FAM-AACGCCTGTGCACGAGCTGAACTAMRA-3' (981-1022).

The qPCR amplification reaction is carried out with One Step PrimeScript RT-PCR Kit (TaKaRa, Dalian, China), and the reaction mixture containing $10 \mu \mathrm{L}$ of $2 \mathrm{X}$ One Step RT-PCR Buffer III, 0.4 $\mu \mathrm{L}$ each of TaKaRa Ex Taq HS $(5 \mathrm{U} / \mu \mathrm{l})$, PrimeScript RT enzyme Mix II, PCR Forward Primer $(0.2 \mu \mathrm{mol} / \mathrm{L})$ and PCR Reverse 
Primer $(0.2 \mu \mathrm{mol} / \mathrm{L}), 0.4 \mu \mathrm{L}$ of Probe $(0.2 \mu \mathrm{mol} / \mathrm{L}), 2 \mu \mathrm{L}$ of total RNA, and then add RNase Free dH2O in a final volume of $20 \mu \mathrm{L}$. The qPCR was conducted with Applied Biosystems 7300FAST Real-Time PCR System under the following conditions: 42 for $5 \mathrm{~min}$ and 95 for $10 \mathrm{sec}, 40$ cycles denaturation at 95 for 5 sec and annealing and elongation at 60 for $20 \mathrm{sec}$, At the end of each annealing and elongation step, fluorescence signals for each sample were collected (Wang et al., 2020). The cycle threshold (CT) values and standard curve were analyzed by Sequence Detector software (version 2.1, Applied Biosystems).

\subsection{Virus isolation and virus replication in cell cultures}

Samples which were detected N-DRV positive were processed for virus isolation using LMH cell, according to the conventional pathogen separation method. We extract the spleen tissues of ducklings and hatching eggs samples, homogenize them by centrifugation, and then freeze and thaw the extracted supernatant three times. The supernatant was filtered through a filter driven by a $0.22 \mu \mathrm{m}$ syringe, and the filtered supernatant was seeded into cell monolayers. Then, the culture was incubated at $37,5 \% \mathrm{CO}_{2}$, and the giant cell or pattern cell lesions were examined daily. When we observed more than $75 \% \mathrm{CPE}$, we collected the virus culture and sub-cultured it until stable CPE could be harvested (Folch et al., 1957). Finally, the cell culture was frozen and thawed three times, and the rest of the study was saved.

2.7 Nucleotide sequencing, sequence and phylogenetic analysis

In this study, phylogenetic analysis was based in the S1 gene of the N-DRV strain. The S1 gene was amplified from the cDNA by RT-PCR with primers N-DRV-F/R, the sequences of the primers were N-DRVF: 5'ATGGATCGCAACGAGGTGATAC-3' (572-593) and N-DRV-R: 5'-CTAGCCCGTGGCGACGGT-3' (1520-1537), which was designed according to N-DRV strain N-DRV-XT18. The PCR products were purified by conventional methods, and then sequenced by BGI Company Ltd (Beijing, China). The sequence was edited using the EditSeq software of the DNASTAR Lasergene 12 Core Suit (DNASTAR, lnc.) The sequence similarities between the isolated strains and published sequences using BLAST online searching program in GenBank (http://blast.ncbi.nlm.nih.gov). We constructed phylogenetic trees of S1 sequences with MEGA V7.0 program using the neighbor-joining method and a bootstrap value selected for 1000 replications (Tang and $\mathrm{Lu}, 2015 \mathrm{a})$.

\section{RESULTS}

\subsection{Serum epidemiological investigation}

Serum samples were collected from 3 farms of breeding duck located in Shandong, Jiangsu and Anhui province, during December 2018 to March 2019. The sampled ducks were all vaccinated with N-DRV vaccine and antibody products, and detection of N-DRV antibody in the samples was performed by indirect ELISA. Of a total of 750 serum samples collected from breeding ducks, the number of N-DRV antibody positive samples was 734, and the positive rate was as high as $97.87 \%$. Among the 119 duckling serum samples, the number of positive samples was 84 , and the positive rate was $70.59 \%$.

\subsection{Clinical signs and pathological features}

following inoculation with N-DRV

Infected breeding ducks were depressed and had decreased appetite. Some breeding ducks developed symptoms of diarrhea, but throughout the experiment, there was no death of infected breeding ducks. Autopsy showed that the main pathological changes in the orally administered and intramuscular injection group were atrophy of follicles (Fig 1A), bleeding of the follicular membrane (Fig 1B), hemorrhage of the fallopian tubes (Fig 1A/B), hemorrhage of the spleen (Fig 1C), and scattered bleeding points in the liver (Fig 1D). In both the orally administered and intramuscular injection group, viral RNA was detected in the ovaries and fallopian tubes of infected ducks.

In the experimental group, the size of embryonated eggs varied (Fig 2A), and the number of inconsistent shape eggs and sand-shelled eggs increased (Fig 2B). During the hatching process, difficulty in pecking the shells occurred (Fig 2D), the embryonic body of the ducklings (Fig 2E), and the liver had obvious bleeding 
symptoms (Fig 2F). The ducklings did not show obvious clinical symptoms except for depressed and lack of appetite (Fig 3G). With the growth of ducklings, necrosis of the spleen of the ducklings revealed several necrotic lesions of varying degrees (Fig $3 \mathrm{H})$. The breeding ducks and hatchling ducklings in the control group were normal, and no pathogenic infection was detected by PCR.

\section{$3.3 \mathrm{~N}-\mathrm{DRV}$ infection reduces fertilization rate and hatchability of breeding ducks}

In this study, a total of 475 hatching eggs were collected for hatching, and hatching was arranged in three times according to the time period, and the fertilization rate and hatching rate were calculated separately. The fertilization rates of the orally administered group, intramuscular injection group and control group were $67.4 \%-70.7 \%, 73.7 \%-80.4 \%$ and $87.3 \%-90.2 \%$, respectively. As for hatching rate, these data were $51.6 \%$ $62.8 \%, 63.6 \%-67.5 \%$ and $92.2 \%-95.7 \%$. The detailed data of the fertilization rate and hatching rate of hatched eggs were shown in Table 1. It can be found that N-DRV infection caused a significant decrease in the fertilization rate and hatching rate of the breeding ducks. Moreover, the fertilization rate and hatching rate of the orally administered group were lower than those of the intramuscular injection group. During the hatching process, embryo death mainly occurred in the middle and late stages of hatching.

\subsection{Positive detection of viral RNA in embryos and ducklings}

101 vitelline membranes, 91 dead embryos and 263 duckling spleen samples were collected in the artificial infection experiment, and qPCR was used to detect viral RNA. Among the samples detected, 18 of 82 yolk membrane samples, 33 of 88 dead embryo samples, and 139 of 141 duckling spleen samples were detected positive for N-DRV. When these two infection methods, the positive rate of viral RNA in the oral administered group was higher than that in the intramuscular injection group. No virus RNA was detected in the control group. Detailed detection data was presented in Table 2.

\subsection{Virus isolation and replication in cell cultures}

Virus isolation results showed that after inoculating homogeneous tissue supernatant into LMH cells, the second-generation cell culture produced CPE in LMH cells within 24 hours after inoculation (Fig. 3A). And nearly $90 \%$ of the cells showed obvious CPE in 72 hours after inoculation (Fig. 3B). The observed CPE was consistent with the classic CPE of ARV, the characteristic giant or bloom-like CPE and the cells fall off in clusters. Based on this, it can be judged that we isolated N-DRV from duck embryos in group. And then, we confirmed the N-DRV positive using RT-PCR amplification. This re-isolated strain was named N-DRV-SD19

\subsection{Sequence comparison and phylogenetic analysis}

The comparison of nucleotide sequence similarity between the N-DRV-SD19 strain and the reference strain (from GenBank) shows that the similarity between N-DRV-SD19 and N-DRV-XT18 was 100\%. The sequence similarities between N-DRV-SD19 and other waterfowl-derived strains isolated in China (except N-DRVXT18) were 96.2-97.8\%, while that with chicken-derived isolates was 37.5-40.6\%, and that with mammalian isolates was 27.1-36.0\%. To investigate the evolutionary relationship between N-DRV-SD19 strain obtained in this study and other Orthoreovirus strains, based on the nucleotide sequence of the S1 gene fragment, we constructed phylogenetic tree using neighbor-joining method (Chen et al., 2020). The analysis results show that SD19 strain was located in in the same evolutionary branch as the novel duck-derived ARV variant N-DRV-XT18 previously reported. In addition, the N-DRV-SD19 was in the same phylogenetic lineage with these strains isolated from Chinese duck flocks in recent years (Fig. 4). It should be noted that although they are both waterfowl-derived strains, the strains isolated in China are far from the D20-99 strain isolated from Hungary.

\section{Discussion}

Since 2017, the prevalence of duck spleen necrosis and swelling has occurred in the main meat duck breeding provinces in China, causing infected ducks growth retardation, the feed return rate drops (Wang et al., 2019), and causing immune suppression to be susceptible to other disease (Montgomery et al., 1986). In previous 
studies, the epidemiological investigation of serum antibody of ducklings found that there was a potential $\mathrm{N}$-DRV infection in the breeding ducks. In order to confirm that N-DRV can be transmitted vertically by breeding eggs, we carried out the artificial infection experiment of breeding ducks.

In the artificial infection test, it can be found that N-DRV infection can cause obvious ovarian atrophy and bleeding, fallopian tube bleeding, and typical spleen swelling and necrosis of the breeding duck, and the fertilization rate and hatching rate of the eggs are significantly reduced. The results of viral RNA detection of Ovary, fallopian tube, egg yolk membrane, duck embryo, duck spleen samples revealed that the N-DRV can spread vertically through the eggs. Although there are still some duck embryos that can be hatched normally, and there are no obvious clinical symptoms in the ducklings, but the spleen of the ducklings shows necrotic lesions of different degrees in the spleen, and the ducklings are not as good as healthy ducks in terms of growth rate and feed return rate (Landman et al., 1994). The damage of immune organs also poses a greater risk for the prevention and control of other epidemics in the later stage (Kibenge et al., 1987). In the comparison of infection routes, the orally admins group has a more significant pathogenic effect than the intramuscular injection group, which is consistent with the main routes of ARVs infection ingesting virus-contaminated feed and drinking water mentioned in previous studies (Landman et al., 1994).

DRV continues to be popular among Chinese ducks, causing various diseases including liver necrosis, splenic necrosis, and hemorrhagic lesions, causing serious economic losses to the Chinese duck farming industry ( $\mathrm{Z}$. Chen et al., 2012; Zheng et al., 2016; Wang et al., 2019). In the previous epidemiological survey, we found that the DRV infection rate of duck flocks in some parts of China (Shandong, Hebei, Jiangxi, Inner Mongolia, Guangxi, Jiangsu, and other provinces) had reached $22.8 \%$, and the DRV infection rate of breeding ducks in some areas exceeds that of meat ducks (Zhang et al., 2013). In another study five years later, it was found that the detection rate of DRV in spontaneous disease ducks reached $38.54 \%$, and the detection rate of DRV in clinically healthy ducks also reached $15.5 \%$, and the co-infection of DRV and other pathogens was widespread (Yang et al., 2018). In terms of seasonal popularity, the incidence of DRV in summer was significantly higher than in other seasons.

In most cases, after the ducks are infected with DRV, these ducks do not show typical symptoms and lesions, and present subclinical infections ( $\mathrm{Li}$ et al., 2016). Only through serological testing can the pathogenic infections be detected. This study found that there were high antibody positive rates in breeding ducks and meat ducks in some areas of China. Since the sampled ducks and meat ducks had not been immunized with $\mathrm{N}$-DRV related vaccine products, it means that N-DRV antibody positive was a natural infection resulting in. This also means that N-DRV infection was already widespread in breeding ducks and meat ducks in some regions, and the infection situation was already very serious. The positive rate of infection of breeding ducks was higher than that of meat ducks, which may be related to factors such as feeding cycle. Infection with ARV reduces the body's immune function, leading to increased susceptibility to other infectious diseases (Kibenge et al., 1987). When vaccinating ducks with other epidemic vaccines, it is easy to cause the immunization effect to fail to reach the standard. Practitioners in the aquaculture industry need to correctly understand and scientifically prevent and control N-DRV infection.

$\mathrm{N}$-DRV is widespread in nature and has strong tolerance to the environment (Heide, 2000). In addition, the virus can spread through horizontal and vertical transmission, making it difficult to prevent and control the disease. Immunization of the breeding ducks is an effective means of controlling the disease, which not only enables the breeding duck population to gain resistance to the virus, but also protects the ducklings through the maternal antibody route and limits the spread of the virus through the vertical transmission route (Menendez et al., 1975). Based on this, the development of vaccines has a very important application value. In addition, the continuous viral shedding in cloaca indicates that feces were non-negligible source of N-DRV infection, prompting us that comprehensive and effective biosecurity measures have a positive effect on the prevention and control of the disease. Carrying out population purification will help reduce or even eliminate the harm of the disease to the duck farming industry.

\section{Acknowledgement}


This study was funded by the National Key Research and Development Program of China (2018YFD05001063; 2018YFD0501506); the China Agriculture Research System (CARS-43-19); the funds of Shandong "Double Top" Program.

\section{Conflict of Interest Statement}

The authors declare that the research was conducted in the absence of any commercial or financial relationship that could be construed as a potential conflict of interest.

\section{Author Contributions}

Conceived and designed the experiments: YD YT HW. Performed the experiments: ZS HW YW BG. Analyzed the data: HW. Contributed reagents/materials/analysis tools: HW ZS. Wrote the paper: HW

\section{Data Availability Statement}

The data that support the findings of this study are available from the corresponding author, Youxiang Diao:yxdiao@163.com; Yi Tang: tyck288@163.com, upon reasonable request.

Figure 1. Pathological changes of breeding ducks caused by N-DRV infection.

(A) The follicles of infected breeding ducks shrank, ovaries bleed, and fallopian tubes bleed; (B) Bleeding follicles and fallopian tubes of infected breeding ducks; (C) Swelling and necrosis of spleen of infected breeding ducks; (D) Infected breeding duck livers were earthy yellow with bleeding spots on the surface.

Figure 2. Duck embryos died during the incubation and the pathological changes in Embryo and ducklings.

(A) The eggs of the experimental group vary in size; (B) The number of inconsistent shape eggs and sandshelled eggs increased; (C) Unfertilized egg in infected group; (D) Some duck embryos had difficulty in breaking shells at the later stage of hatching; (E) Severe bleeding of embryo body; (F) Dead duck embryo with hemorrhage in liver, (1) Infected group duck embryo and (2) control duck embryo; (G) Some ducklings were depressed; (H) Spleen of duckling presents with severe splenic necrosis.

Figure 3. CPE in N-DRV-SD19 infected LMH cells. (A) The second generation of LMH cells showed CPEs $24 \mathrm{hr}$ after infection; (B) The second generation of LMH cells showed CPEs $72 \mathrm{hr}$ after infection; (C) Non-infected LMH cell $24 \mathrm{hr}$ after test; (D) Non-infected LMH cell $72 \mathrm{hr}$ after test.

Figure 4. Phylogenetic tree constructed based on nucleotide sequences of S1 genome segment by Orthoreovirus species.

\section{References}

Chen, H, Y. Tang, Y. Dou, X. Zheng, and Y. Diao, 2016: Evidence for Vertical Transmission of Novel Duck-Origin Goose Parvovirus-Related Parvovirus. Transboundary and emerging diseases 63 , 243-247, DOI: $10.1111 /$ tbed.12487.

Chen, Hao, M. Yan, Y. Tang, and Y. Diao, 2019: Pathogenicity and genomic characterization of a novel avian orthoreovius variant isolated from a vaccinated broiler flock in China. Avian Pathology 48, 334-342, DOI: $10.1080 / 03079457.2019 .1600656$.

Chen, Hao, M. Yan, Y. Tang, and Y. Diao, 2020: Sequencing and phylogenetic analysis of partial S1 genes of avian orthoreovirus isolates in Shandong province during 2015-2017. Poultry science99 , 2416-2423, DOI: 10.1016/j.psj.2019.11.067.

Chen, Z., Y. Zhu, C. Li, and G. Liu, 2012: Outbreak-associated Novel Duck Reovirus, China, 2011. Emerging Infectious Diseases $\mathbf{1 8 ,} 1209$.

Folch, J., M. Lees, and G.H.S. Stanley, 1957: A simple method for isolation and purification of the total lipids from animal tissues. J.biol.chem 226 , 497-509. 
Heide, L. Van Der, 2000: The History of Avian Reovirus. Avian Diseases 44, 638-641.

Joklik, W.K., 1982: Structure and function of the Reovirus genome.Microbiological reviews 45 , 483-501.

Jones, R.C., 2000: Avian reovirus infections. Revue scientifique et technique (International Office of Epizootics) 19, 614-625, DOI: 10.20506/rst.19.2.1237.

Jones, R.C., and J.R.M. Guneratne, 1984: The pathogenicity of some avian reoviruses with particular reference to tenosynovitis. Avian Pathology 13, 173-189.

Kibenge, F.S.B., R.C. Jones, and C.E. Savage, 1987: Effects of experimental immunosuppression on reovirusinduced tenosynovitis in light-hybrid chickens. Avian Pathology Journal of the W.v.p.a16, 73-92.

Landman, W.J., E. Gruys, and R.M. Dwars, 1994: A syndrome associated with growth depression and amyloid arthropathy in layers: a preliminary report. Avian pathology : journal of the W.V.P.A 23, 461470, DOI: $10.1080 / 03079459408419016$.

Li, N., T. Hong, Y. Wang, Y. Wang, K. Yu, Y. Cai, S. Liu, L. Wei, and T. Chai, 2016: The pathogenicity of novel duck reovirus in Cherry Valley ducks. Veterinary microbiology 192, 181-185, DOI: 10.1016/j.vetmic.2016.07.015.

Malkinson, M., K. Perk, and Y. Weisman, 1981: Reovirus infection of young Muscovy ducks (Cairina moschata). Avian pathology : journal of the W.V.P.A 10 , 433-440, DOI: 10.1080/03079458108418493.

Menendez, N.A., B.W. Calnek, and B.S. Cowen, 1975: Experimental egg-transmission of avian reovirus. Avian diseases 19 , 104-111.

Menendez, N.A., and B.W.C.S. Cowen, 1975: Localization of avian reovirus (FDO isolant) in tissues of mature chickens. Avian Diseases 19, 112-117.

Montgomery, R.D., P. Villegas, D.L. Dawe, and J. Brown, 1986: A Comparison between the Effect of an Avian Reovirus and Infectious Bursal Disease Virus on Selected Aspects of the Immune System of the Chicken.Avian Diseases 30 , 298-308.

Nick, H., D. Cursiefen, and H. Becht, 1975: Structural and growth characteristics of two avian reoviruses. Archives of Virology 48, 261.

Su, Y.P., B.S. Su, J.H. Shien, H.J. Liu, and L.H. Lee, 2006: The sequence and phylogenetic analysis of avian reovirus genome segments $\mathrm{M} 1, \mathrm{M} 2$, and M3 encoding the minor core protein muA, the major outer capsid protein muB, and the nonstructural protein muNS. Journal of Virological Methods 133, 146-157.

Tang, Y., and H. Lu, 2015a: Genomic characterization of a broiler reovirus field strain detected in Pennsylvania. Infection Genetics \& Evolution 31, 177-182.

Tang, Y., and H. Lu, 2015b: Genomic characterization of a novel avian arthritis orthoreovirus variant by next-generation sequencing. Archives of Virology 160, 2629-2632.

Tang, Y., H. Lu, A. Sebastian, Y.T. Yeh, C.A. Praul, I.U. Albert, and S.Y. Zheng, 2015: Genomic characterization of a turkey reovirus field strain by Next-Generation Sequencing. Infection Genetics $\mathcal{E}$ Evolution 32, 313-321.

Vilmos, P., G. Róbert, D.K. Mihály, I. Eva, N. Er?Sné, B. Krisztián, R. Gábor, S. Gy?Rgy, D. Adám, and B. Mária, 2003: Reovirus identified as cause of disease in young geese. Avian Pathology Journal of the W.v.p.a $32,129-138$.

Vindevogel, H., G. Meulemans, P.P. Pastoret, A. Schwers, and C.M. Calberg-Bacq, 1982: Reovirus infection in the pigeon. Annales De Recherches Veterinaires Annals of Veterinary Research 13 , 149.

Wang, H., B. Gao, H. Chen, Y. Diao, and Y. Tang, 2019: Isolation and characterization of a variant duck orthoreovirus causing spleen necrosis in Peking ducks, China. Transboundary and Emerging Diseases DOI: 
10.1111/tbed.13252.

Wang, H., B. Gao, X. Liu, S. Zhang, Y. Diao, and Y. Tang, 2020: Pathogenicity of a variant duck orthoreovirus strain in Cherry Valley Ducklings. Veterinary microbiology 242 , 108546, DOI: 10.1016/j.vetmic.2019.108546.

Wei, F., J. Yang, D. He, Y. Diao, and Y. Tang, 2020: Evidence of vertical transmission of novel astrovirus virus in goose. Veterinary microbiology 244, 108657, DOI: 10.1016/j.vetmic.2020.108657.

Yang, J., R. Zhang, Z. Wang, X. Yu, X. Niu, Y. Tang, and Y. Diao, 2018: Epidemiological investigation on co-infection of duck viral epidemic diseases in some areas of my country. Chinese Journal of Veterinary Medicine 38 , 1871-1877.

Yun, T., W. Ye, Z. Ni, L. Chen, B. Yu, J. Hua, Y. Zhang, and C. Zhang, 2012: Complete Genomic Sequence of Goose-Origin Reovirus from China.Journal of Virology 86 , 13143.

Zhang, S., J. Yang, Z. Wang, L. Chen, Y. Diao, and Y. Tang, 2020: Development of an ELISA to distinguish between goose parvovirus infection and vaccine immunization antibodies. Poultry science99 , 1332-1340, DOI: $10.1016 /$ j.psj.2019.12.012.

Zhang, Y., Y. Diao, X. Song, X. Ju, C. Yu, and P. Pei, 2013: Investigation on co-infection of pathogens of immunosuppressive diseases in commercial meat ducks. Chinese Journal of Veterinary Medicine 33(06):828$832+837$.

Zheng, X., D. Wang, K. Ning, T. Liang, M. Wang, M. Jiang, and D. Zhang, 2016: A duck reovirus variant with a unique deletion in the sigma $\mathrm{C}$ gene exhibiting high pathogenicity in Pekin ducklings. Virus Research $215,37-41$.

\section{Hosted file}

Table.docx available at https://authorea.com/users/344363/articles/470931-evidence-ofvertical-transmission-of-novel-duck-orthoreovirus-in-ducks
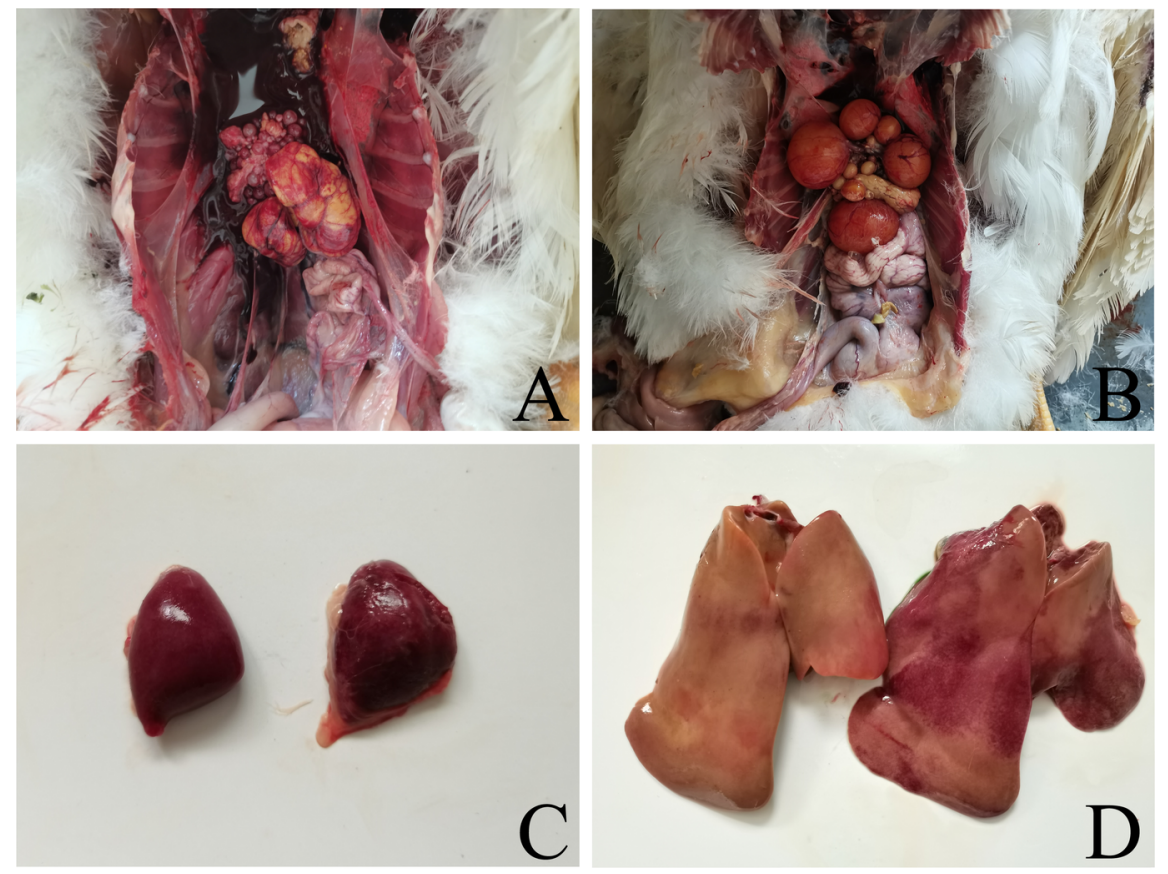

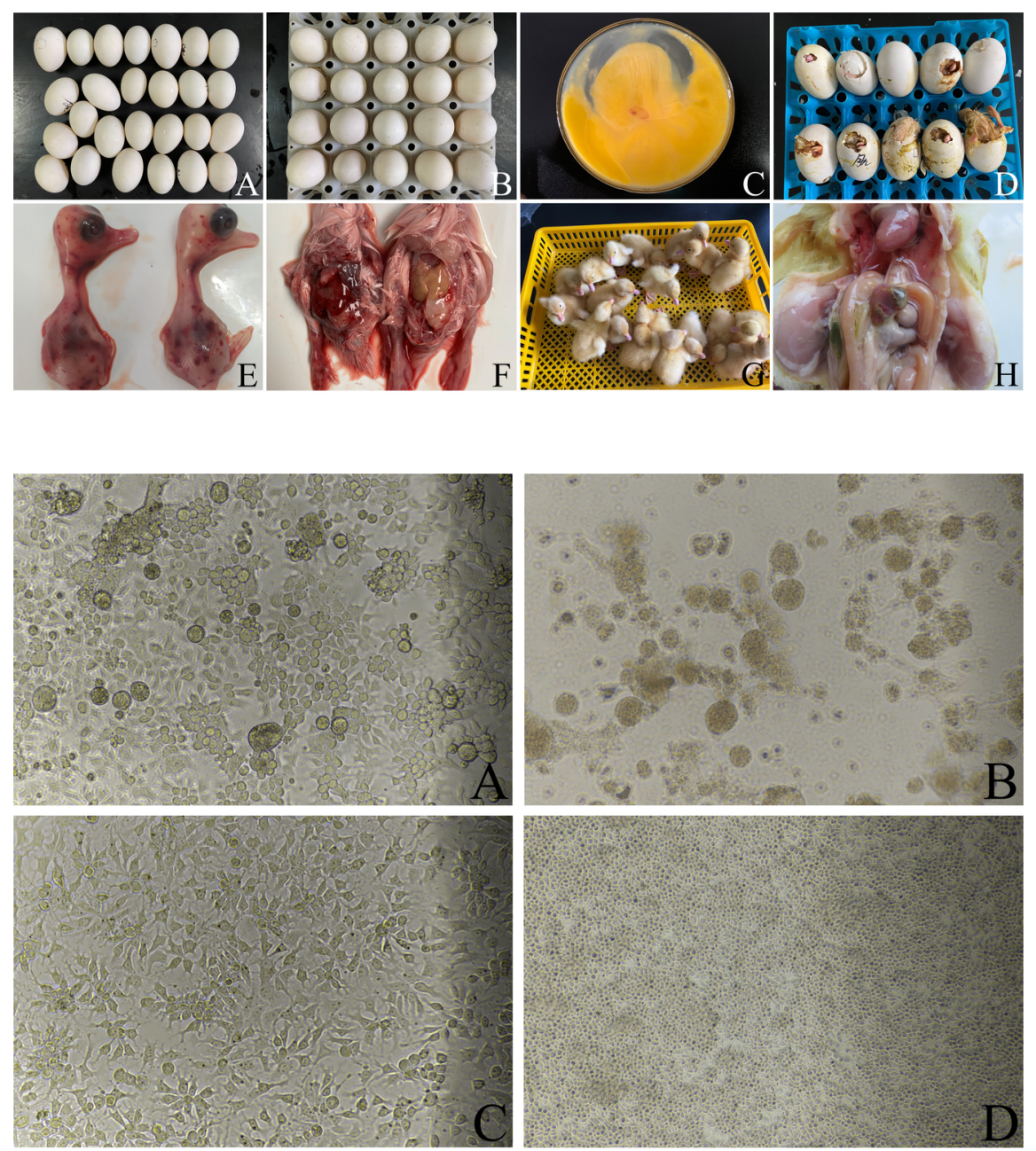


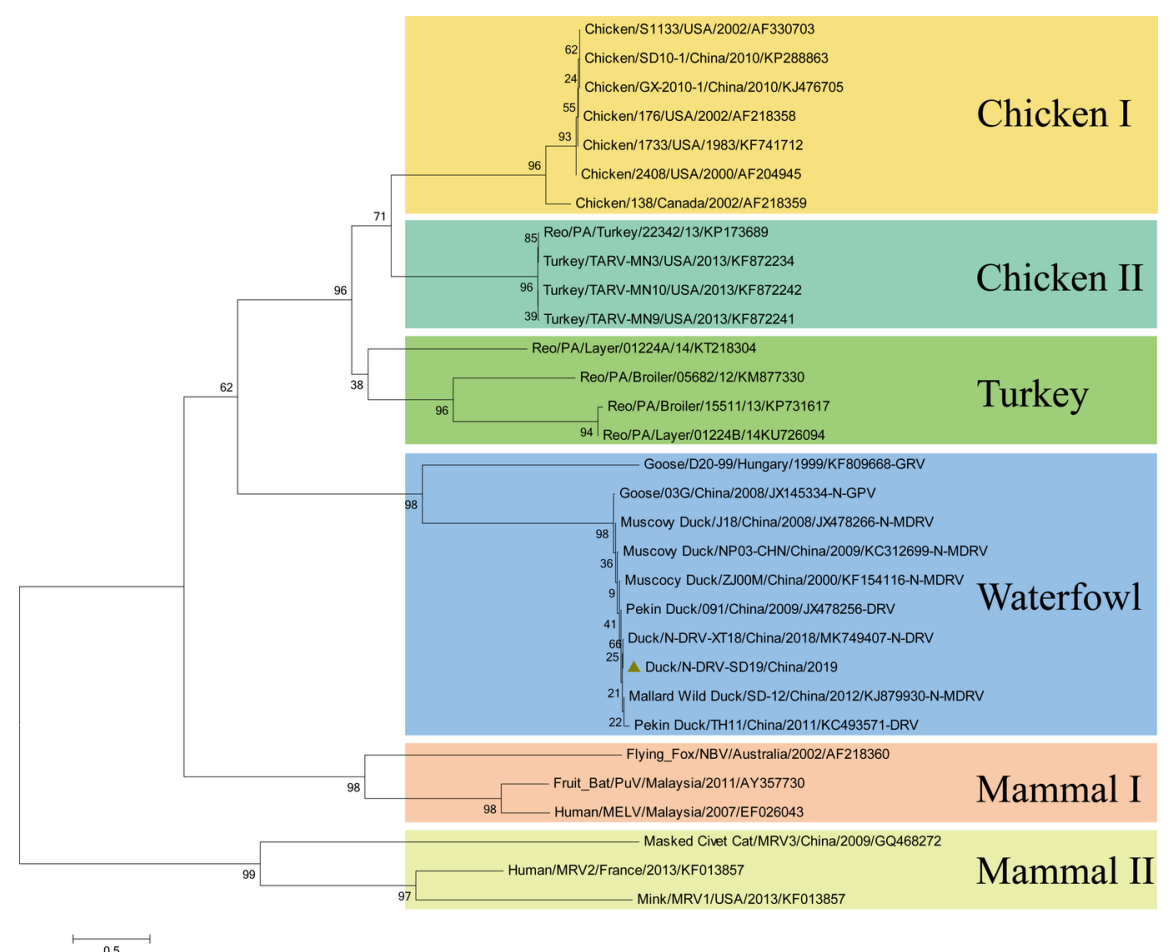

